\title{
Relazioni e interferenze tra acquiferi profondi e superficiali
}

Francesco La Vigna

LinQ - Laboratorio di Idrogeologia Numerica e Quantitativa

Università degli Studi Roma Tre - Roma

lavigna@uniroma3.it

Nello studio dei sistemi idrogeologici, le relazioni esistenti tra acquiferi profondi e superficiali giocano sempre un importante ruolo per la definizione degli schemi concettuali di circolazione regionale. A causa della profondità, il primo tipo di acquifero risulta spesso facilmente in comunicazione con fluidi endogeni, con masse magmatiche in raffreddamento (Taran et al. 1998) e in generale maggiormente influenzato dal flusso di calore e dal generale gradiente geotermico locale (Capasso et al. 2001). Tutto ciò tende a modificare lo stato fisico e chimico delle acque presenti in questi serbatoi che possono ricevere gli apporti di ricarica in settori di affioramento ristretti o dove le condizioni geologico-strutturali permettano un collegamento idraulico con gli acquiferi superficiali. Il secondo tipo di acquifero, quello freatico superficiale riceve generalmente i suoi apporti di ricarica maggiori dalle precipitazioni, dall'alimentazione dei corsi d'acqua e da eventuali apporti laterali. Gli scambi tra acquiferi profondi e superficiali possono avvenire sia dall'alto verso il basso che viceversa (Brusca et al., 2001); questi rapporti sono sempre strettamente connessi all'assetto geologico-strutturale regionale che a luoghi genera delle vie di fuga (Shukla et al., 2010) delle acque e dei fluidi profondi verso l'alto, o delle direttrici principali di ricarica verso il basso. In alcuni casi le relazioni sono anche causate dall'intervento antropico per mezzo di opere di captazione o esplorazione mal realizzate (Humez et al., 2011).

Nella letteratura internazionale e nazionale recente gli studi che affrontano la tematica relativa agli scambi e alle miscelazioni tra acquiferi affrontano la problematica con le metodologie della geochimica isotopica e che riguardano spesso la caratterizzazione geotermica di reservoir profondi (Carucci et al., 2012). Le prospezioni geochimiche delle acque sotterranee infatti rappresentano un metodo di indagine che consente di ricostruirne i circuiti (Rodgers et al., 2005), di caratterizzarne il bacino di alimentazione di risalire alle temperature alle quali sono state in equilibrio in profondità (Aquilina et al., 2002) e di evidenziare fenomeni di mescolamento (Chae et al., 2006).

Tramite questi studi che utilizzano gli elementi maggiori e in traccia disciolti e gli isotopi di vari elementi come traccianti naturali, infatti, si riesce a determinare con buona approssimazione l'origine delle acque. Gli isotopi maggiormente utilizzati in queste analisi sono gli isotopi Ossigeno-18 e Deuterio, il Trizio e il Carbonio $\left({ }^{13} \mathrm{C}\right)$. Gli isotopi di Ossigeno e Deuterio permettono di seguire il percorso dell'acqua nei vari momenti del ciclo idrologico, dalla formazione delle masse di vapore sopra gli oceani alla loro condensazione e precipitazione, permettono inoltre di risalire alla latitudine e all'altitudi- ne di infiltrazione; il Trizio è presente in atmosfera dall'inizio degli esperimenti nucleari e permette quindi datazioni relative agli ultimi 50 anni, mentre il Carbonio permette di descrivere tutti i rapporti di interazione tra fasi gassose, liquide e solide che presiedono all'acquisizione del carico salino delle acque. L'IAEA (International Atomic Energy Agency), ha un attivo settore (www.iaea.org/water) dedicato allo studio e alla ricerca sull'idrologia isotopica e pubblica anche un periodico sull'argomento (Newsletter of the Isotope Hydrology Section ISNN 1020-7120).

Nell'ambito della ricerca delle relazioni tra acquiferi profondi e superficiali risultano invece meno frequenti gli studi di carattere idrogeologico-quantitativo, cioè quegli studi che cercano di capire le relazioni tra i diversi acquiferi analizzando i termini del bilancio idrologico (Cardenas e Wilson, 2006) in relazione all'assetto geologico-strutturale delle rocce serbatoio (Join et al., 2005). In questi studi vengono effettuate numerose misure piezometriche, di flusso delle sorgenti e dei deflussi in alveo dei corsi d'acqua, vengono monitorati sia agli acquiferi superficiali che quelli profondi (La Vigna et al., 2012) così come vengono valutati i prelievi antropici per poi essere messi a confronto con i dati relativi agli apporti meteorici nelle aree di ricarica dei bacini idrogeologici individuati (Chiocchini et al., 2010; Mazza e La Vigna, 2011). Anche quando la profondità e le condizioni di pressione e temperatura lo permettono il monitoraggio degli acquiferi profondi è piuttosto difficile da mettere in opera, sia perché le perforazioni profonde attrezzate a fori di monitoraggio sono piuttosto rare, sia perché a causa della loro frequente artesianità, il loro monitoraggio prevede strumenti di misura particolari, come flange di testa pozzo con manometri, torri piezometriche e sonde multiparametriche adatte a resistere in queste condizioni. Grande importanza in questi studi hanno le prospezioni geofisiche, sia sismiche che geoelettriche, che permettono la ricostruzione dei serbatoi e delle loro caratteristiche con buona approssimazione (Tronicke e Holliger, 2005).

Se da una parte gli studi chimici ed isotopici portano quanto alla definizione dell'origine e ai tempi di residenza delle acque nei serbatoi profondi (Kloppmann et al., 1998), dall'altra la loro spiccata vocazione geochimica a volte, tende a non prendere in piena considerazione le geometrie dei bacini idrogeologici e i rapporti idraulici tra le idrostrutture (Andre et al., 2005). Allo stesso modo gli studi puramente quantitativi spesso mancano di approfondimenti geochimici ed isotopici che possano avvalorare i percorsi delle acque individuati. La best practice per individuare i processi di miscelazione e le relazioni tra diversi acquiferi risulta quindi essere un approccio 
multidisciplinare che studi i rapporti isotopici e geochimici delle acque alla luce però delle geometrie degli acquiferi, delle loro relazioni idraulico-geometriche individuate e dei volumi scambiati calcolati (Petitta et al., 2011; Portugal et al., 2005)

\section{BIBLIOGRAFIA}

Andre L., Franceschi A., Pouchan P., Atteia O. (2005) - Using geochemical data and modelling to enhance the understanding of groundwater flow in a regional deep aquifer, Aquitaine Basin, south-west of France. Journal of Hydrology 305: 40-62 DOI 10.1016/j.jhydrol.2004.08.027

Aquilina L., Ladouche B., Doerfliger N., Seidel JL., Bakalowicz M., Dupuy C., Le Strat P. (2002) - Origin, evolution and residence time of saline thermal fluids (Balaruc springs, southern France): implications for fluid transfer across the continental shelf. Chemical Geology 192: 1-21 DOI Pii s0009-2541(02)00160-2

10.1016/s0009-2541(02)00160-2

Brusca L., Aiuppa A., D’Alessandro W., Parello F., Allard P., Michel A. (2001) - Geochemical mapping of magmatic gas-water-rock interactions in the aquifer of Mount Etna volcano. Journal of Volcanology and Geothermal Research 108: 199-218 DOI 10.1016/ s0377-0273(00)00286-9

Capasso G., D’Alessandro W., Favara R., Inguaggiato S., Parello F. (2001) - Interaction between the deep fluids and the shallow groundwaters on Vulcano island (Italy). Journal of Volcanology and Geothermal Research 108: 187-198 DOI 10.1016/s03770273(00)00285-7

Cardenas MB., Wilson JL. (2006) - The influence of ambient groundwater discharge on exchange zones induced by current-bedform interactions. Journal of Hydrology 331: 103-109 DOI 10.1016/j. jhydrol.2006.05.012

Carucci V., Petitta M., Aravena R. (2012) - Interaction between shallow and deep aquifers in the Tivoli Plain (Central Italy) enhanced by groundwater extraction: A multi-isotope approach and geochemical modeling. Applied Geochemistry 27: 266-280 DOI 10.1016/j.apgeochem.2011.11.007

Chae GT., Yun ST., Kim K., Mayer B. (2006) - Hydrogeochemistry of sodium-bicarbonate type bedrock groundwater in the Pocheon spa area, South Korea: water-rock interaction and hydrologic mixing. Journal of Hydrology 321: 326-343 DOI 10.1016/j.jhydrol.2005.08.006
Chiocchini U., Castaldi F., Barbieri M., Eulilli V. (2010) - A stratigraphic and geophysical approach to studying the deep-circulating groundwater and thermal springs, and their recharge areas, in $\mathrm{Ci}-$ mini Mountains-Viterbo area, central Italy. Hydrogeology Journal 18: 1319-1341 DOI 10.1007/s10040-010-0601-5

Humez P., Audigane P., Lions J., Chiaberge C., Bellenfant G. (2011) Modeling of $\mathrm{CO}(2)$ Leakage up Through an Abandoned Well from Deep Saline Aquifer to Shallow Fresh Groundwaters. Transport in Porous Media 90: 153-181 DOI 10.1007/s11242-011-9801-2

Join JL., Folio JL., Robineau B. (2005) - Aquifers and groundwater within active shield volcanoes. Evolution of conceptual models in the Piton de la Fournaise volcano. Journal of Volcanology and Geothermal Research 147: 187-201 DOI 10.1016/j.jvolgeores.2005.03.013

Kloppmann W., Dever L., Edmunds WM. (1998) - Residence time of chalk groundwaters in the Paris Basin and the North German Basin: a geochemical approach. Applied Geochemistry 13: 593-606 DOI 10.1016/s0883-2927(97)00110-8

La Vigna F., Carucci V., Mariani I, Minelli L., Pascale F., Mattei M., Mazza R., Tallini M. (2012) - Intermediate-field hydrogeological response induced by L'Aquila earthquake: the Acque Albule hydrothermal system (Central Italy). Italian Journal of Geosciences 131

Mazza R., La Vigna F. (2011) - Hydrogeology of the southern Middle Tiber Valley (Central Italy). AQUA mundi 2: 93-102 DOI 10.4409/Am-031-11-0031

Petitta M., Primavera P., Tuccimei P., Aravena R. (2011) - Interaction between deep and shallow groundwater systems in areas affected by Quaternary tectonics (Central Italy): a geochemical and isotope approach. Environmental Earth Sciences 63: 11-30 DOI 10.1007/ s12665-010-0663-7

Portugal E., Izquierdo G., Truesdell A, Alvarez J. (2005) - The geochemistry and isotope hydrology of the Southern Mexicali Valley in the area of the Cerro Prieto, Baja California (Mexico) geothermal field. Journal of Hydrology 313: 132-148 DOI 10.1016/j.jhydrol.2005.02.027

Rodgers P., Soulsby C., Waldron S., Tetzlaff D. (2005) - Using stable isotope tracers to assess hydrological flow paths, residence times and landscape influences in a nested mesoscale catchment. Hydrology and Earth System Sciences 9: 139-155

Shukla R., Ranjith P., Haque A., Choi X. (2010) - A review of studies on $\mathrm{CO}(2)$ sequestration and caprock integrity. Fuel 89: 2651-2664 DOI 10.1016/j.fuel.2010.05.012

Taran Y., Fischer TP., Pokrovsky B, Sano Y., Armienta MA., Macias JL. (1998) - Geochemistry of the volcano-hydrothermal system of El Chichon Volcano, Chiapas, Mexico. Bulletin of Volcanology 59: 436-449 DOI 10.1007/s004450050202

Tronicke J., Holliger K. (2005) - Quantitative integration of hydrogeophysical data: Conditional geostatistical simulation for characterizing heterogeneous alluvial aquifers. Geophysics 70: H1-H10 DOI $10.1190 / 1.1925744$ 